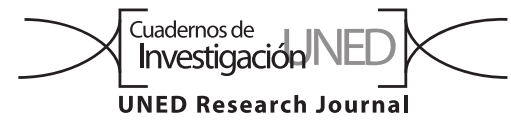

\title{
El género Liocanthydrus en Venezuela (Coleoptera: Noteridae) con descripción de dos nuevas especies
}

\author{
Mauricio M. García R. ${ }^{1}$, Jesús A. Camacho M. ${ }^{2}$ \& Nedy C. Poleo S. ${ }^{2}$ \\ 1. Centro de Investigaciones Biológicas (CIB), Facultad de Humanidades y Educación, edificio de postgrado, Universidad del Zulia. Apdo. 526, \\ A-4001. Maracaibo, Estado Zulia, Venezuela; meruidae2014@gmail.com \\ 2. Museo de Artrópodos de la Universidad del Zulia (MALUZ). Departamento Fitosanitario, Facultad de Agronomía, Universidad del Zulia. Apar- \\ tado 15205. Maracaibo, ZU 4005. Venezuela; jcamacho@fa.luz.edu.ve; nedypoleo@fa.luz.edu.ve
}

Recibido 02-XI-2017 • Corregido 15-II-2018 • Aceptado 27-III-2018

\begin{abstract}
The genus Liocanthydrus in Venezuela (Coleoptera: Noteridae) with description of two new species. The genus Liocanthydrus contains rare species, as they are not abundant and lack records to indicate their distribution. The genus has undergone several taxonomic changes, from its first status as a subgenus to the current genus status. Two species of Liocanthydrus (Noteridae: Noterini) are described. Liocanthydrus ramosae sp. nov. and L. distintus sp. nov., both collected manually, in two freshwater microhabitats, from the Aguaro and Orinoco rivers, in the plains of Venezuela. Habits of the present species in Venezuela and the genitals structures of the two species described are included. A key is constructed to identify the species Liocanthydrus.
\end{abstract}

Key words: aquatics coleopterous, hercircum, new specie, Plains of Venezuela, ramentum.
RESUMEN: El género Liocanthydrus contiene especies raras, por ser poco abundantes y carecer de registros que indiquen su distribución. El género ha sufrido varios cambios taxonómicos, desde su primer estatus como subgénero hasta su estatus genérico actual. Se describen dos especies de Liocanthydrus (Noteridae: Noterini). Liocanthydrus ramosae sp. nov. y L. distintus sp. nov., ambas colectadas de forma manual, en dos microhábitats de agua dulce, de los ríos Aguaro y Orinoco, en los llanos de Venezuela. Se incluyen habitus de las especies presentes en Venezuela y las estructuras genitales de las dos especies descritas. Se construye una clave para identificar las especies de Liocanthydrus.

Palabras clave: Coleópteros acuáticos, hercircum, nueva especie, Planicies de Venezuela, ramentum.
El género Liocanthydrus Guignot, 1957 pertenece a la familia Noteridae, tribu Noterini. Las especies de este género son raras, por la poca abundancia y distribución que se registra en las colectas. El género ha sufrido varios cambios taxonómicos, desde su primer estatus como subgénero hasta su estatus genérico actual (Miller, 2009; Baca, Gustafson, Toledo \& Miller, 2014). Las procedencias de las especies de Liocanthydrus, ha sido focalizada a Brasil, Paraguay y Venezuela.

Guignot erigió el género Liocanthydrus, como un subgénero de Canthydrus Sharp, 1882 en la especie $C$. (Liocanthydrus) angustus Guignot, 1957, de Brasil. Laporte describió una nueva especie C. (Liocanthydrus) buqueti (Laporte, 1835) de Guyana, la cual, posteriormente fue renombrada en un nuevo género Canthysellus (Baca \& Toledo, 2015). Zimmermann describió dos especies más C. (Liocanthydrus) octoguttatus (Zimmermann, 1921), y C. (Liocanthydrus) uniformis (Zimmermann, 1921), de Brasil, para un total de tres especies. Liocanthydrus fue elevado a la categoría de género por Miller (2009). Baca et al. (2014), revisaron el género describiendo dos nuevas especies L. armulatus (Baca et al., 2014), de Venezuela y L. nanops (Baca et al., 2014), de Paraguay, sinonimizando las especies de Siolius clayae (Balfour-Browne, 1969), y L. bicolor (Balfour-Browne, 1969), sumando a siete el número de especies para el género, ya que S. amazonensis (Balfour-Browne, 1969), fue sinonimizado con L. uniformis (Zimmermann, 1921). Finalmente, Guimarães and Ferreira-Jr. (2015), describieron dos nuevas especies de Brasil, L. zanclus y L. mecespilus (Guimarães \& Ferreira-Jr., 2015), para un total de nueve especies en el género.

El objetivo fundamental de la investigación fue la descripción de dos nuevas especies de Liocanthydrus en Venezuela. Ambas especies fueron colectadas en dos 
sistemas hidroecológicos, Morichal y Limnico (García, Vera, Benetti \& Blanco-Belmonte, 2016), y junto a L. clayae y $L$. armulatus suman cuatro las especies presentes en Venezuela y aumenta a once el número de especies para el género.

\section{MATERIALES Y MÉTODOS}

Material estudiado: Se examinaron 27 ejemplares (incluyendo holotipos y ejemplares de $L$. armulatus y $L$. clayae), colectados en dos localidades entre el municipio Pedro Camejo en el estado Apure y municipio Las Mercedes en el estado Guárico, Venezuela. Las especies fueron colectadas manualmente en pequeños cuerpos de agua sobre los corredores ribereños de los ríos Orinoco y Aguaro. El material se encuentra depositado en la colección del Museo de Artrópodos de La Universidad del Zulia, Maracaibo, Zulia, Venezuela.

Identificación taxonómica y extracción de órganos genitales: El reconocimiento de las especies se realizó utilizando un estereomicroscopio marca Leica Wild M10 de alta resolución, ocular $80 x$ y objetivos 25x. Algunos ejemplares estuvieron sometidos a un tratamiento con agua caliente durante dos minutos, para producir el ablandamiento de los escleritos genitales. Seguidamente fueron extraídos en solución de glicerina absoluta, luego sumergidos en $\mathrm{KOH}$ al 10\%, durante varios min para aclararlos y eliminar restos de tejido conectivo adheridos a la quitina. Las genitalias de los machos se dibujaron con una cámara lúcida y diseñada con Corel Draw Graphi Suite X8 versión 1.0.298.1. Las estructuras genitales se preservaron en micro viales, con solución alcohólica de glicerina, para posteriores usos.

Las claves sistemáticas que se construyen para introducir las dos nuevas especies de Liocanthydrus son una adaptación de Baca et al. (2014) y Guimarães y Ferreira-Jr. (2015). Se presenta un mapa con las localidades de las especies de Liocanthydrus en Venezuela (Fig. 3).

\section{RESULTADOS}

\section{Tratamiento taxonómico:}

Familia Noteridae Thomson, 1860

Subfamilia Noterinae Thomson, 1860

Tribu Noterini Thomson, 1860

Género Liocanthydrus Guignot, 1957

Liocanthydrus ramosae

García, Camacho \& Poleo sp. nov. (Fig. 1A)
Diagnosis. Tres pares de mancha dorsales: un primer par de manchas alargadas amarillentas, sobre los márgenes laterales del pronoto, ancha en el extremo anterior y aguzándose hacia la parte posterior; un par de manchas amarillentas de diámetro mayor sobre la región humeral del primer tercio elitral en cada élitro y un par de manchas amarillentas de menor diámetros sobre el disco central del primer tercio elitral y dos pares sobre el tercio apical de diámetros similar a las manchas del primer tercio elitral (Fig. 1A).

Material tipo. Holotipo $\sigma^{7}$, Venezuela, Apure Pedro Camejo, (Morichal 6017'30,01"N 67028'40,18”W, 64m), 12.i.2006, M. García col. (MALUZ 05802). 2 Paratipos $0^{7}$, con los mismos datos del holotipo (MALUZ 0580305804). 4 Paratipos 우, con los mismos datos del holotipo (MALUZ 05805-05808).

Descripción. Longitud 2,8mm y ancho 1,3mm. Forma oval alargada con el extremo anterior redondeado con su mayor anchura en el primer tercio elitral, aguzándose hacia el último tercio apical elitral. Coloración de la cabeza y pronoto castaño y una banda irregular testácea amarillenta sobre los márgenes laterales del pronoto. Élitros castaño rojizo obscuro con un par de manchas o máculas amarillentas, sobre el primer y segundo tercio elitral. Ventralmente testáceo con márgenes y suturas castaño obscuro.

Cabeza con superficie microreticulada y puntuaciones escasas y muy espaciadas. Margen anterior del clípeo con una hilera de micro puntos y la base antenal con una hendidura lateral. Antenas con antenómeros moderadamente largos y cilíndricos con un par de seta en el margen preapical (una a cada lado). Último antenómero apical de menor longitud a los dos penúltimo antenómeros que antecede, con el ápice aguzado. Palpómero maxilar terminal largo, delgado, con el ápice finamente aplanado con una fina hendidura que separa a dos sensores terminales. Último palpómero labial largo, ancho y aguzado en el ápice, con el margen anterior cóncavo o curvado en la mitad basal y recta en la mita apical con una protuberancia cilíndrica y corta o sensor, en la mitad. Margen posterior convexo. Gula con superficie con pequeñas y finas estría transversales. Pronoto con superficie finamente puntuada, de puntos muy espaciada. Margen anterior con hilera de puntos a lo largo del margen. Margen lateral ancho, con una línea impresa submarginal completa y continúa hasta el margen angular anterior. Disco elitral con superficie microreticulada y micropuntuada espaciadamente. Margen elitral lateral moderadamente ancho. Prosterno de márgenes laterales ancho. Disco prosternal ancho con el margen anterior gruesamente marginado, con una muy leve 

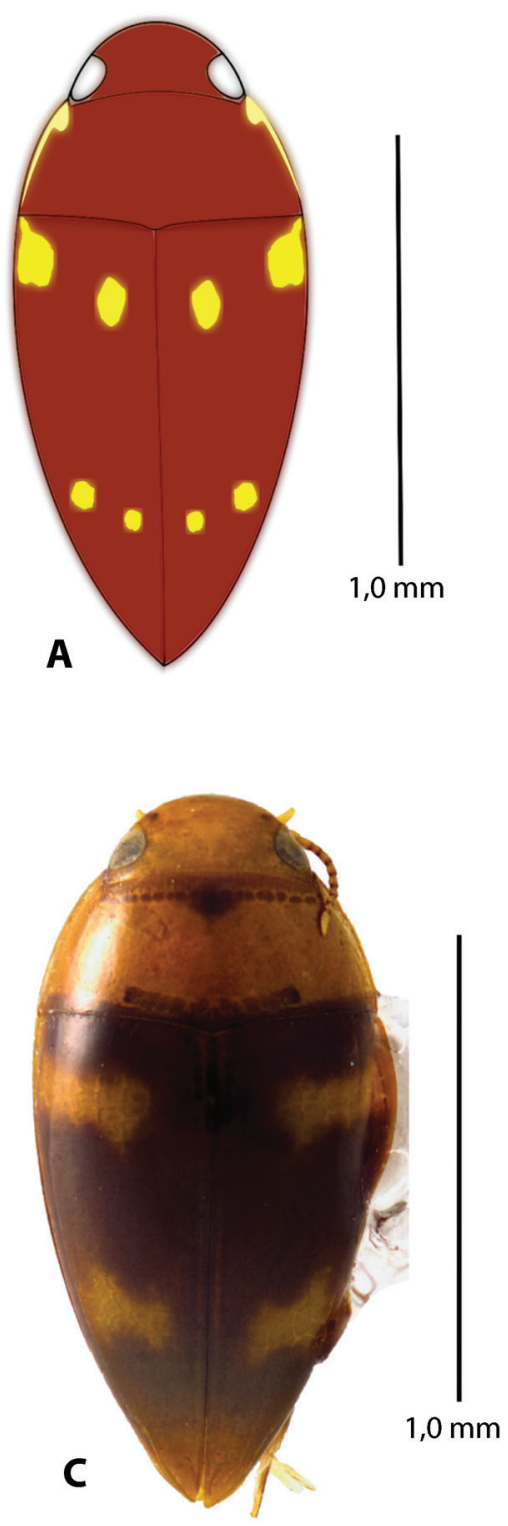
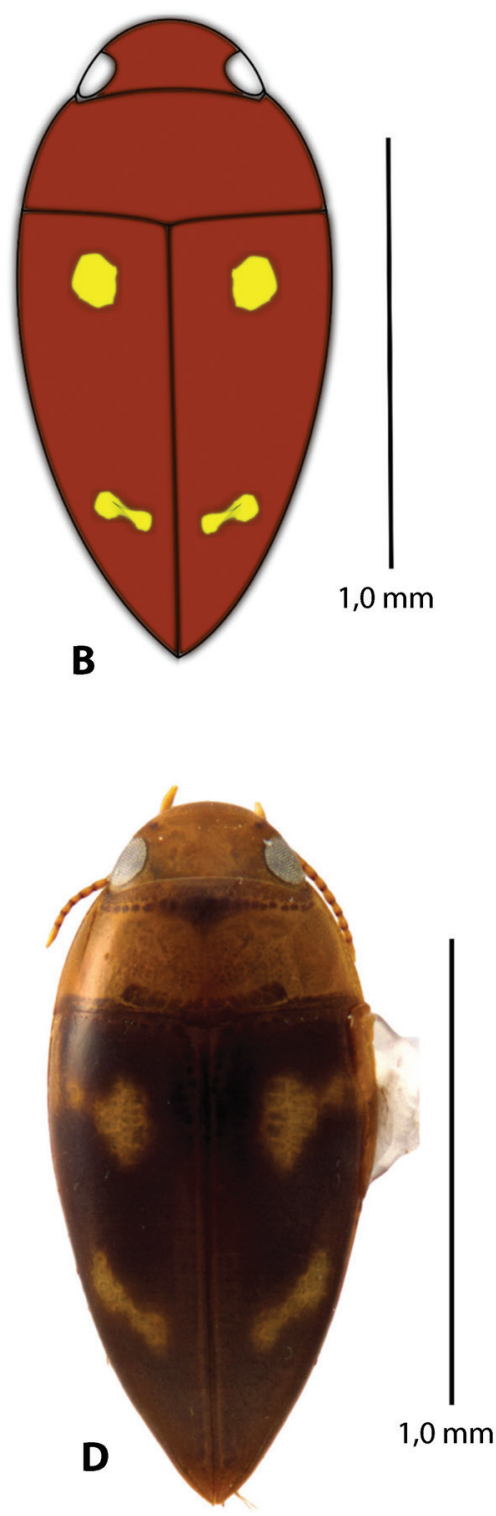

Fig. 1. Habitus. A) Liocanthydrus ramosae sp. nov. B) Liocanthydrus distintus sp. nov. C) Liocanthydrus armulatus y D) Liocanthydrus clayae (Tomado de Baca et al., 2014).

protuberancia medial. Apófisis prosternal de márgenes (lateral y apical) gruesos y sinuoso. Superficie claramente microreticulada. Metasterno de márgenes ancho. Placa noteroide de superficie claramente reticulado y margen posterior sinuoso con la placa coxal ancha, superficie microreticulada, y lóbulos coxales fuertemente angulados con tres setas gruesas en el ápice. Coxas anteriores anchas y globulares, coxas medias cubiertas bajo la placa metasternal. Trocánter anterior y medio anchos y cortos, trocánter posterior casi la mitad de la longitud del fémur. Fémur anterior ancho y superficie lisa y pulida, con una hilera de setas moderadas en el margen preapical posterior. Tibia anterior delgada y casi la mitad de la longitud del fémur. Margen anterior recto y margen posterior moderadamente curvo con una hilera de pequeñas setas o espinas bordeándolo hasta el margen apical. $1^{\text {er }}$ tarsómero largo y grueso., $2^{\text {do }}$ tarsómero corto con una ventosa basal, $3^{\text {er }}$ tarsómero cortos y expandidos combinados casi la mitad de la longitud del primero, con una ventosa basal, $4^{\text {to }}$ segmento muy corto y $5^{\text {to }}$ segmento largo y curvo de casi la longitud del primero con dos uñas apicales. Una espuela o espolón moderadamente robusto, 
largo y curvo en el margen apical. Fémur medio similar al fémur anterior. Tibia media dos tercios de la longitud del fémur, ancho y rectangular, con cinco espinas gruesas (una basal muy corta y cuatro largas, espaciadas por aproximadamente su longitud) sobre el margen posterior. Margen anterior con ocho espinas. Margen posterior con cuatro espinas largas (una muy corta y tres moderadas y separadas aproximadamente por su longitud). Margen apical con una corona de espinas moderadas. Abdomen con la superficie pulida en cada esternito, último esternito convexo. Estructura genital (Fig. 2A)
Hembra. Longitud $2,9 \mathrm{~mm}$ y ancho $1,3 \mathrm{~mm}$ Similar en forma y coloración al macho, diferenciándose en la ausencia de ventosas en los tarsos anterior y medio.

Variación. Los ejemplares colectados presentan variación en las proporciones dimensionales entre machos y hembras, variando en longitud entre 2,7 y $2,9 \mathrm{~mm}$ y ancho entre 1,2 y 1,3mm, así como en la forma de las manchas elitrales, las cuales nunca se presentan simétricas o de la misma dimensión, entre machos y hembras y entre machos y entre hembras.
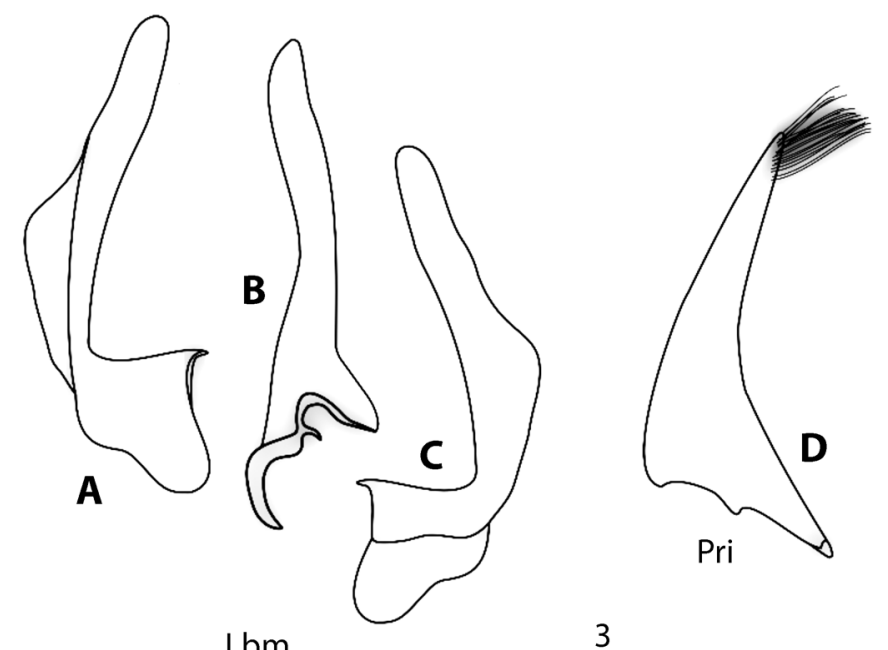

3

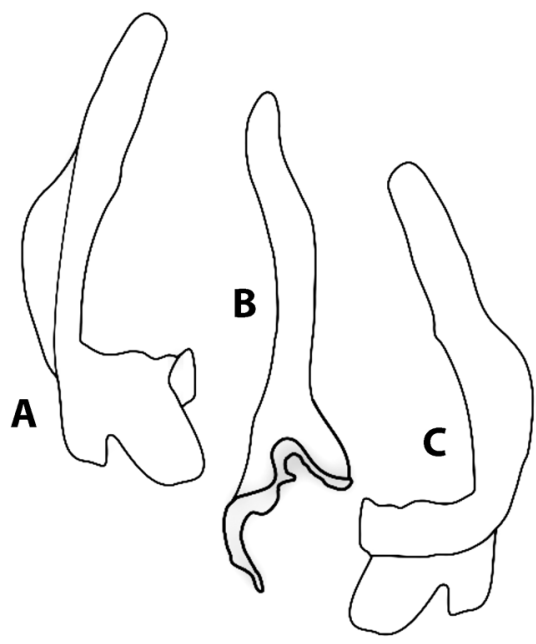

Lbm

4
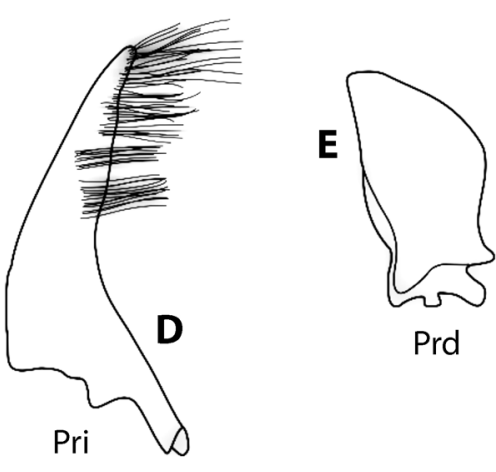

Prd

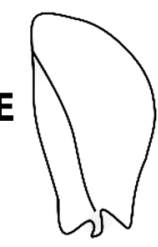

Prd

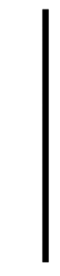

Fig. 2. Organos genitales de a) Liocanthydrus ramosae sp. nov y b) Liocanthydrus distintus sp. nov. Lbm= lóbulo medio: A) vista lateral izquierda, B) vista ventral y C) vista lateral derecha, Pri= parámetro izquierdo y Prd= parámetro derecho. 


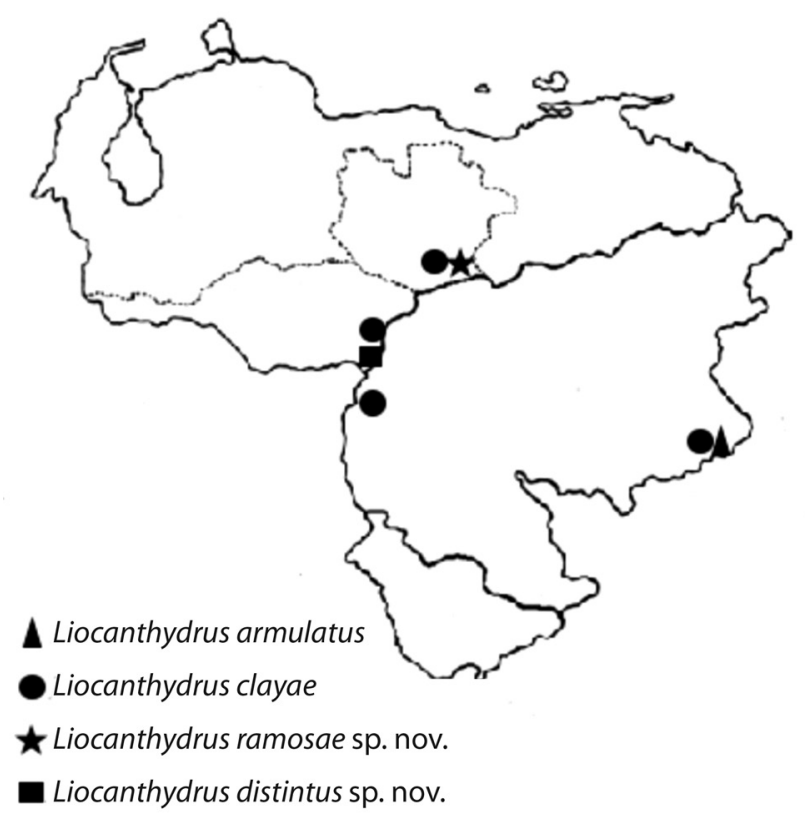

Fig. 3. Distribución de las especies de Liocanthydrus en Venezuela.

Etimología. La especie se dedica a Noris Margarita Ramos, mi esposa.

Habitalogía. El hábitat característico de esta especie es el hercircum con abundantes plantas Juncaceae, superpuestas sobre un sustrato ramentum, en un sistema hidroecológico del tipo Morichal/limnico (García et al., 2016).

Distribución. La especie se restringe a la localidad de captura, en la región sur de los llanos, en el estado Apure, Venezuela.

\section{Liocanthydrus distintus \\ García, Camacho \& Poleo, sp. nov. (Fig. 1B)}

Diagnosis. Similar L. ramosae sp. nov., en tamaño y forma. Se diferencia por la posición de las manchas amarillentas sobre el dorso elitral que son más reducidas y menos apreciables y la ausencia de una banda lateral sobre el margen pronotal, presente en L. ramosae.

Material tipo. Holotipo $\diamond$, Venezuela, Guárico, Las Mercedes, (Río Aguaro, Estación Experimental NicolasitoUCV 8०09'25,01"N 66²6'42,92"O, 52 m), 19.i.2009, M. García col. (MALUZ 05787). 6 Paratipos ơ, con los mismos datos del holotipo (MALUZ 05796-05801). 8 Paratipos 우, con los mismos datos del holotipo (MALUZ 05788-05795).
Descripción. Longitud 3,0mm y ancho 1,4mm. Forma oval alargada, con el margen anterior redondeado, su mayor anchura en el tercio basal elitral y aguzado hacia la tercia apical elitral. Coloración de la cabeza y pronoto castaño, y élitros de castaño rojizo obscuro, con dos pares de manchas testáceo amarillento sobre el primer y segundo tercio apical. Ventralmente amarillento con márgenes y suturas castaño rojizo.

Cabeza con superficie microreticulada y pulida, con puntuaciones escasas muy espaciadas. Margen anterior del clípeo con una serie de micro puntuaciones. Base antenal con hendidura lateral. Antenas con antenómeros cortos y emarginados latero apicalmente, con un par de setas pequeñas, una a cada lado en el margen preapical. Último segmento o antenómero terminal largo y aguzado apicalmente, de longitud a la equivalente de los dos penúltimos combinados. Palpo maxilar con el último palpómero largo y delgado, casi la longitud de los tres que le anteceden. Ápice aplanado y hendido en el borde. Palpos labiales con los dos primeros palpómeros cortos un quinto de la longitud del tercero. Tercer palpómero largo y ancho con margen anterior curvo en su mitad basal y recta en su mitad apical, con un sensor cilíndrico en la mitad y margen posterior convexo, ápice agudo. Gula ancho y superficie pulida. Pronoto microreticulado y pulido, con finas puntuaciones muy espaciadas. Margen anterior con una hilera de finísimos puntos con micro setas a lo largo del margen. Margen lateral completo (línea submarginal se extiende hasta el margen anterior, por encima del ángulo lateral) y ancho. Disco elitral microreticulado, pulida y brillante con líneas o estrías longitudinales impresas bajo el integumento. Margen lateral moderadamente ancho. Prosterno con márgenes gruesamente emarginados. Disco prosternal ancho, con el margen anterior gruesamente emarginado con una pequeña protuberancia aguda medialmente. Apófisis prosternal de márgenes (lateral y apical) gruesos y sinuosos y superficie lisa y pulida. Superficie central y basal de la apófisis prosternal con una estrella de cinco puntas impresa bajo el integumento. Metasterno largo y márgenes anchos. Placa noteroide metasternal ancho de lados casi paralelos, levemente divergentes. Superficie lisa y pulida, margen posterior sinuoso. Placa noteroide con lóbulos coxales largos con tres setas gruesas en cada ápice. Coxas anteriores anchas y globulares, coxas medias cubiertas bajo la placa metasternal. Trocánter anterior y medio anchos y cortos, trocánter posterior casi la mitad de la longitud del fémur. Fémur anterior ancho y superficie lisa y pulida, con una hilera de setas moderadas en el margen preapical posterior. Tibia anterior delgada y casi la mitad de la longitud del fémur. Margen anterior recto y margen posterior moderadamente curvo con una 
hilera de pequeñas setas o espinas bordeándolo hasta el margen apical. 1er tarsómero largo y grueso., 2do tarsómero corto con una ventosa basal, 3er tarsómero cortos y expandidos combinados casi la mitad de la longitud del primero, con una ventosa basal, 4to segmento muy corto y 5 to segmento largo y curvo de casi la longitud del primero con dos uñas apicales. Una espuela o espolón moderadamente robusto, largo y curvo en el margen apical. Fémur medio similar al fémur anterior. Tibia media dos tercios de la longitud del fémur, ancho y rectangular, con cinco espinas gruesas (una basal muy corta y cuatro largas, espaciadas por aproximadamente su longitud) sobre el margen posterior. Margen anterior con ocho espinas. Margen posterior con cuatro espinas largas (una muy corta y tres moderadas y separadas aproximadamente por su longitud). Margen apical con una corona de espinas moderadas. Esternito abdominales de superficie pulida, con el último segmento convexo. Estructura genital (Fig. 2B)

Hembra. Longitud 2,9mm y ancho $1,3 \mathrm{~mm}$, de aspecto similar al holotipo, pero con cierta opacidad sobre las manchas dorso elitrales. Ventralmente hay ausencia de ventosas de adhesión en los dos primeros tarsómero de las patas anteriores y medias.

Variación. La longitud varía entre 2,8 y $2,9 \mathrm{~mm}$ y el ancho entre 2,3 y $2,4 \mathrm{~mm}$, en cuanto a las manchas sobre la superficie pronotal y elitral, siendo en las hembras algo más expandidas que en los machos.

Etimología. Esta especie es muy similar a L. ramosae, pero claramente es observable la forma distinta que presentan las manchas amarillas sobre el dorso elitral, por esta razón se escoge un adjetivo como epíteto para esta especie.

Habitalogía. Esta especie fue colectada en un sistema hidroecológico limnico/lótico/léntico (García et al., 2016) en dos microhábitats superpuestos, hercircum-ramentum. Charcas originadas por desbordes del rio, con ribera arenosa y hierbas vasculares acuáticas, en el corredor ribereño del río Aguaro.

Distribución. La especie se encuentra restringida a la localidad de la colecta por el corredor ribereño del río Aguaro, región llanera del estado Guárico, Venezuela.

\section{Liocanthydrus armulatus \\ Baca, Gustafson, Toledo y Miller (2014) (Fig. 1C)}

Localidad tipo. Venezuela, Bolívar, El Paujil.

Descripción simple. Forma oval alargada, aguzado hacia el extremo apical de los élitros. Longitud 3,0 $\mathrm{mm}$ y ancho $1,5 \mathrm{~mm}$. Aspecto dorsal muy similar a L. clayae. Cabeza pequeña con ojos pequeños, pronoto con margen lateral ancho, élitros con márgenes moderado y ascendente en vista lateral.

Habitalogía. La especie tiene un atractivo por el ramentum y el hercircum dos tipos de microhábitats, presentes tanto en el sistema Morichal como en el sistema limnico (García et al., 2016).

Distribución. Estado Bolívar.

$$
\begin{gathered}
\text { Liocanthydrus clayae } \\
\text { J. Balfour-Browne (1969) (Fig. 1D) }
\end{gathered}
$$

Siolius clayae J. Balfour-Browne (1969: 5) (orig. descr. Guyana); Nilsson (2005: 124) (cat.); Nilsson (2011: 31) (cat.).

Liocanthydrus clayae (J. Balfour-Browne, 1969); Baca, Gustafson, Toledo \& Miller (2014: 241).

Localidad tipo. Guyana, Montaña de Kanuku, Rupununi.

Descripción simple. Forma oval alargada aguzada en el extremo apical de los élitros. Largo entre 2,7 y 3,0mm y ancho entre 1,2 y 1,3mm. Coloración castaño amarillento en la cabeza y pronoto y castaño rojizo obscuro en los élitros y bandas transversales castaño amarillento a amarillo en los élitros. Cabeza con ojos grandes, pronoto con margen lateral ancho, élitros con márgenes laterales delgados.

Habitalogía: La especie tiene un atractivo por el ramentum y el hercircum dos tipos de microhábitats, presentes tanto en el sistema Morichal como en el sistema limnico (García et al. 2016).

Distribución. Estados Amazonas, Apure, Bolívar y Guárico.

\section{Comentario taxonómico}

Las cuatro especies presentan casi una similitud dorsal. Todas presentan manchas impresas transversalmente entre castaño amarillenta y amarillentas sobre los élitros. Las especies $L$. ramosae sp. nov., presenta una banda latero longitudinal clara en el pronoto y en vez de bandas presenta manchas acirculares amarillentas en los élitros y L. distintus sp. nov., no presenta una banda pronotal pero igualmente presentan manchas acirculares y una irregular banda preapical en los élitros. La estructura de los órganos genitales es muy variada en las cuatro especies 
y determinante en la separación de las once especies. Los lóbulos medio de todas las especies, presentan un arco lateral, que se extiende en los dos tercios apicales de la estructura lobular, la cual varia en su extensión, claramente observable en las once especies, y en la forma y estructura de los parámeros, donde es mayor el resalte de los escleritos (Baca et al., 2014; Guimarães \& Ferreira-Jr., 2015).

\section{Claves para separar las especies de Liocanthydrus Guignot, 1957}

[Modificado y adaptado de Baca et al. (2014) y Guimarães y Ferreira-Jr. (2015)]

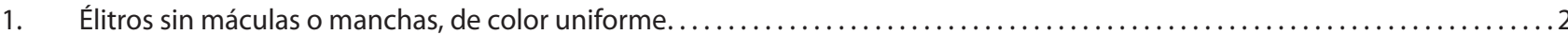

$1^{\prime} \quad$ Élitros y pronoto, maculado en manchas irregulares, puntos reducidos o bandas, de color amarillo a rojo o marrones ......3

2. Forma robusta; distancia intraocular más de dos tercios del ancho de la cabeza; armadura genital con el parámero izquierdo corto, aproximadamente dos tercio de la longitud del edeago (Baca et al., 2014); Brasil ................... L. uniformis

Forma oval y delgada; distancia intraocular menor de dos tercios del ancho de la cabeza; armadura genital con el parámero izquierdo largo, delgado, casi tan largo como el edeago (Baca et al., 2014); Brasil y Guayana Holandesa ........ L. angustus

3. Élitros con maculas amarillas a bandas de color marrón rojizo, que se extienden transversalmente, desde los márgenes laterales, casi hasta la comisura elitral, o proyectadas débilmente, de forma oblicua, rara vez reducidas a manchas...........4

3' Máculas en los élitros y pronoto que consiste en manchas o bandas orientadas longitudinal u oblicuamente, o puntos amari-

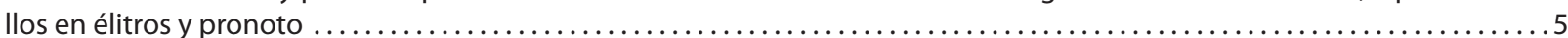

4. Armadura genital con el edeago extendido distalmente, redondeado en el ápice; parámero izquierdo con una franja de pelos restringida a la mitad distal; parámero derecho, triangular, con el ángulo distal claramente redondeado, no proyectado (Baca et al., 2014); Brasil..

L. bicolor

4' Armadura genital con el edeago débilmente expandido distalmente, antes de la atenuación de ápice redondeado; parámero izquierdo, con una franja de pelos que se extiende a lo largo del margen dorsal interior hasta la mitad de su longitud; parámero derecho, sub triangular, emarginado moderadamente a lo largo de la longitud del margen dorsal, ángulo distal proyectándose con ápice redondeado (Baca et al., 2014); Venezuela................................ L. armulatus

5. Máculas en el disco elitral, con grandes bandas longitudinales u oblicuas en la mitad basal, extendiéndose hasta la mitad apical de los élitros, o en su defecto orientadas lateralmente, reducido a menudo a pequeñas manchas en la mitad de los élitros.

Máculas en pronoto y élitros con manchas y pequeños puntos amarillo................................ 10

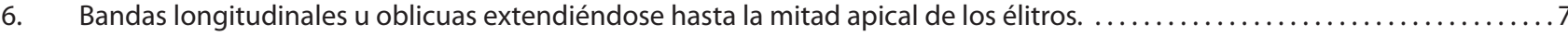

6' Manchas laterales en la mitad de los élitros

7. Élitros con cuatro máculas: una pequeña mancha o banda cerca del ángulo humeral; un punto grande y ancho, longitudinal en la mitad basal elitral; un punto pequeño en la mitad apical elitral, cerca de la comisura elitral; una banda muy grande, alargada oblicuamente que se extiende hacia abajo del margen lateral, cerca del ápice; edeago ventralmente sin rebordes laterales; parámero izquierdo sin flecos o pelos (Guimarães \& Ferreira-Jr., 2015); Brasil....

L. zanclus

7' Élitro con tres máculas: una pequeña mancha o banda cerca del ángulo humeral; una banda grande y oblicua en la mitad basal elitral; una banda alargada que se extiende oblicuamente desde el margen lateral hasta la comisura elitral, cerca del ápice; ventralmente armadura genital con rebordes laterales en el edeago; parámero izquierdo con una franja de flecos o pelos (Guimarães \& Ferreira-Jr., 2015); Brasil .... L. mecespilus

8. Ojos grandes; maculas en los élitros, con bandas posteriores o manchas que se extiende antero lateralmente casi hasta la mitad basal de los élitros; armadura genital con el edeago esbelto sin aspecto sinuoso dorsalmente; débilmente expandido anteriormente, atenuando gradualmente alargando el ápice; parámero izquierdo delgado; parámero derecho triangular y sin proyección del ángulo distal (Baca et al., 2014); Guyana, Surinam y Venezuela ........................... clayae

Ojos pequeños; bandas posteriores o puntos se extienden distalmente más allá de la mitad elitral, desde el disco central hacia

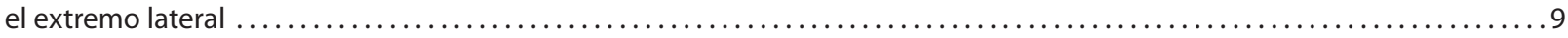

9. Armadura genital con el edeago corto distalmente y atenuado en la cara lateral; parámero izquierdo ancho; parámero derecho sub triangular, con proyección del ángulo distal (Baca et al., 2014); Brasil y Paraguay 
Armadura genital con el edeago alargado distalmente y márgenes subparalelos; parámero izquierdo menos amplio y distalmente atenuado; parámero derecho triangular con ángulo agudo distal, no proyectado (Baca et al., 2014); Brasil

L. Octoguttatus

10. Pronoto con una banda marginal longitudinal amarillo testáceo y una mancha reducida a un punto amarillo sobre el disco pronotal lateral; élitros con una mancha grande amarilla orientada lateralmente y una mancha amarilla de menor dimensión sobre el disco central, en el primer tercio elitral; dos manchas amarillas reducidas a puntos orientadas oblicuamente, sobre el último tercio elitral; Venezuela. .

L. ramosae sp. nov.

10' Pronoto impecable; disco central elitral con una pequeña mancha circular amarilla en el primer tercio elitral; mancha oblicua amarilla reducida en la mitad, sobre el tercio apical elitral; Venezuela

L. distintus sp. nov.

\section{DISCUSIÓN}

El género Liocanthydrus Guignot, en los últimos 17 años, ha experimentado un incremento en su estudio, ya que, durante un tiempo, este género fue poco estudiado. Las especies del género no son muy comunes, en su mayoría, son encontradas en coexistencia con especies de otros géneros como Hydrocanthus Say, 1823, Canthysellus y Suphisellus Crotch, 1873. Liocanthydrus ramosae sp. nov. y L. distintus sp. nov., ambas fueron colectadas en la región sur de Venezuela. Liocanthydrus ramosae fue colectado en un ambiente moriche (García et al., 2016), en Apure y L. distintus en un ambiente formado por pequeños cuerpos de agua producto de inundaciones, en la estación experimental Nicolasito-UCV, en los llanos centrales del estado Guárico. Ambas especies son muy similares externamente, pero se diferencian de las otras nueve especies al comparar los órganos genitales. Dorsalmente, los élitros presentan una variación en las máculas o manchas, muy apreciables con respecto a las otras especies. De todas las especies descritas anteriormente, solo L. ramosae, presenta una banda pronotal marginal.

\section{AGRADECIMIENTOS}

Agradezco a Luis José Joly por invitarme a la estación de San Nicolasito de la UCV y a Manuel Echenique por su valiosa colaboración en la logística y apoyo vehicular, en los Ilanos de Apure y Guárico.

\section{REFERENCIAS}

Baca, S. M., Gustafson, G. T., Toledo, M., \& Miller, K. B. (2014). Revision of the Neotropical burrowing water beetle genus Liocanthydrus Guignot (Coleoptera: Noteridae: Noterinae: Noterini) with the description of two new species. Zootaxa, 3793(2), 231-246. doi:10.11646/ zootaxa.3793.2.3

Baca, S. M., \& Toledo, M. (2015). Canthysellus Baca \& Toledo (Coleoptera: Noteridae: Noterini), a New Genus of Burrowing Water Beetle from South America. The Coleopterists Bulletin, 69(3), 477-488. doi:10.1649/0010-065X-69.3.477

Balfour-Browne, J. (1969). A new genus of Noteridae (Coleoptera: Noteridae: Noterini). Proceedings of the Royal Entomological Society of London, Series B (Taxonomy), 38 (1-2), (5-6). doi:10.1111/j.1365-3113.1969.tb00215.x

García, M., Vera, A., Benetti, C. J., \& Blanco-Belmonte, L. (2016). Identificación y clasificación de los microhábitats de agua dulce. Acta Zoologica mexicana, 32(1), 12-31. doi:10.21829/azm.2016.3201923

Guignot, F. (1957). Contribution à la connaissance des dytiscides Sud-Américains. Revue Française d'Entomologie, 24 33-45.

Guimarães, B. A., \& Ferreira-Jr. N. (2015). Two new species and new records of Liocanthydrus Guignot (Coleoptera: Noteridae) from Brazil. Zootaxa, 3914(5), 591-596. doi:10.11646/zootaxa.3914.5.8

Miller, K. B. (2009). On the Systematics of Noteridae (Coleoptera: Adephaga: Hydradephaga): Phylogeny, description of a new tribe, genus and species, and survey of female genital morphology. Systematics and Biodiversity, 7, 191-214. 